\title{
Historia y usos del adverbio pronto*
}

\author{
History and uses of the adverb pronto \\ Francisco Javier Herrero Ruiz de Loizaga \\ Instituto Universitario Menéndez Pidal, Universidad Complutense de Madrid. \\ Correo electrónico: fjavierh@ucm.es
}

\begin{abstract}
Estudiamos en este artículo el desarrollo de los usos de pronto, adverbio que en el español actual es el más frecuente para la expresión de la cercanía temporal. Sin embargo, su empleo con este valor es relativamente reciente. Pronto, cultismo de escaso empleo introducido a finales de la Edad Media (indicio de ello es que todavía no lo recoge Covarrubias a principios del XVII), era un adjetivo que presentaba el valor de 'presto, aparejado, disponible', y que posteriormente irá utilizándose en usos adverbiales que indican ya no la disposición para la acción sino la realización en breve plazo de la acción. El paso a los usos adverbiales, acompañado de una importante caída de los usos adjetivos, se consuma en el siglo XVIII (el Diccionario de Autoridades todavía no recoge el uso como adverbio), y crece de un modo notable en el XIX, desplazando a presto, que con anterioridad había sido el adverbio de mayor uso en esta función.
\end{abstract}

Palabras clave: adverbio temporal, cercanía temporal, adverbialización, español moderno.

In this article we study the development of the uses of pronto, the Spanish adverb most frequently used to express temporal proximity. Nevertheless, this is a relatively recent use. Pronto was in its origin a learned word of limited use which was introduced at the end of the Middle Ages (evidence of it is the fact that the word is not included in the Covarrubias dictionary at the beginning of the $17^{\text {th }}$ century). It was an adjective that had the meaning of 'ready, prompt, prepared'. It would later have adverbial uses, which no longer show disposition for action, but the fulfilment of the action in a short period of time. In the $18^{\text {th }}$ century, pronto acquires its adverbial uses (the Diccionario de Autoridades still doesn't include its adverbial values). In the same period of time, there is also an obvious decline of the adjective uses. In the $19^{\text {th }}$ century, the use of pronto increases in a considerable way, replacing presto, which was before the most common adverb for the expression of temporal proximity.

Key words: adverbs of time, temporal proximity, adverbialization, modern Spanish.

Este trabajo se inscribe en el marco del proyecto de investigación FFI2015-64080-P, Procesos de gramaticalización en la historia del español (V): gramaticalización, lexicalización y análisis del discurso desde una perspectiva histórica, del Ministerio de Economía y Competitividad. 


\section{INTRODUCCIÓN}

En este trabajo nos proponemos estudiar el desarrollo del adverbio temporal pronto, que se enmarca dentro del grupo que la Real Academia (RAE-ASALE 2009: 2316) denomina "adverbios referenciales". Hoy en día, pronto es el adverbio más frecuente para la expresión de la cercanía temporal (sin señalamiento explícito de inmediatez temporal, como muestran otros adverbios referenciales como en seguida/ enseguida), aunque no se agoten aquí los valores que este adverbio puede presentar en español. Hay que hacer notar, sin embargo, que el empleo de pronto con este valor temporal es relativamente reciente: su uso es ajeno al español medieval y clásico, en que funciona como adjetivo, y ha llegado a los usos actuales tras un proceso de cambio funcional que conduce a su adverbialización - motivado en gran medida por la influencia de otro elemento parcialmente análogo, como es presto-y tras un progresivo, aunque aún no total, abandono de sus originales empleos adjetivos.

La situación que encontramos en el español medieval y clásico respecto al uso de los adverbios que indican cercanía o inmediatez temporal es completamente distinta a la actual, y el elenco de adverbios medievales para la expresión de los valores que ahora estudiamos es a su vez bastante distinto del que había en latín.

En el español medieval, los adverbios utilizados para la expresión de la cercanía o inmediatez temporal eran, siguiendo la nómina que proporciona Sánchez Lancis (1990: 317), luego con el valor de 'enseguida', y aína, presto, pri(v)ado, y cedo con el valor de 'pronto', aunque en este último grupo de adverbios no es siempre fácil delimitar el valor de mera cercanía temporal y el de inmediatez'. De estos adverbios solo cedo 'pronto, rápidamente' $(<\mathrm{CITO})$, procede directamente de un adverbio latino. Los demás se han creado mediante diversos procedimientos de adverbialización. Corominas, al que sigue en este punto Sánchez Lancis (1990: 318), señala que cedo tuvo vitalidad hasta el siglo XIV, para quedar luego reducido al lenguaje popular o arcaizante. Los datos del CORDE y el $\mathrm{CDH}^{2}$ siguen reflejando el uso de cedo hasta el siglo XVI, y de un modo muy residual todavía en el XVII; pero ya en el siglo XVI aparece con especial intensidad en colecciones de refranes o libros de caballerías, como el Primaleón, que tienen un estilo especialmente arcaizante. Pri(v)ado, que alcanza su uso adverbial probablemente a partir de su primitivo uso adjetivo ${ }^{3}$, tiene

También pueden presentar un valor adverbial de manera, 'rápidamente'. Para los usos celerativos de estos adverbios v. Rodríguez Molina (2014: 751-757).

2 El CORDE (Corpus Diacrónico del Español) recoge documentación desde los orígenes de la lengua hasta 1974. El CDH (Corpus del Nuevo Diccionario Histórico del Español) recoge textos desde los orígenes al año 2000, y está basado en los corpus de CREA (Corpus de referencia del español actual) y CORDE. Por ello, los ejemplos que proporciona el CDH hasta 1974 son casi siempre coincidentes con CORDE. El CDH ha sido sometido a un proceso de lematización que le da mayor flexibilidad para realizar algunos tipos de consultas. No obstante, en función de lo que se quiera buscar y cómo, puede ser preferible el uso de un corpus u otro, por lo que en este trabajo emplearemos ambos. Es irrenunciable el empleo de CORDE para un estudio diacrónico. Como señala la propia Academia, el CORDE es hoy "fuente obligada para cualquier estudio diacrónico relacionado con la lengua española. La Academia utiliza sistemáticamente el CORDE para documentar palabras, para calificarlas de anticuadas o en desuso, para saber el origen de algunos términos, su tradición en la lengua, las primeras apariciones de las palabras...” (http://www.rae. es/recursos/banco-de-datos/corde).

3 El uso adverbial de privado no es de fácil explicación y se han propuesto diversas etimologías. Meyer Lübke (apud Corominas 1980-1991, vol. IV: 655b) entendía que era préstamo del occitano abrivat, -ada 'rápido, impetuoso', derivado de briu, 'brío', hipótesis que Corominas (1980-1991, vol. IV: 655b) considera "defendible", siempre que se acepte una alteración por etimología popular que lo relacionaría con privado al entrar en castellano; pero prefiere 
cierto empleo hasta el siglo XIV (Sánchez Lancis, 1990: 318); aína, procedente de un sustantivo latino vulgar AGĪNA, 'actividad, prisa', derivado de AGĔRE 4 , fue el adverbio más frecuente con el valor de 'pronto' en el español medieval, pero va siendo sustituido por presto en el siglo XV. No obstante, aína aún tiene cierto empleo en el Siglo de Oro, aunque va perdiendo prestigio frente a presto. Covarrubias (s. v. ayna) todavía afirma que es palabra muy usada, pero ya Autoridades dice que "se usa vulgarmente" . Presto, que a partir de su originario empleo adjetivo había alcanzado usos adverbiales ya en el siglo XIII, probablemente a través de empleos como predicativo ${ }^{6}$, se mantendrá como el adverbio más usado para el valor de cercanía temporal a lo largo del Siglo de Oro e incluso en el siglo XVIII, no siempre netamente desligado del de posterioridad inmediata, 'enseguida'. También luego sigue siendo el principal adverbio usado con el valor de 'enseguida' en el español clásico e incluso en el siglo XVIII.

\section{APARICIÓN DE PRONTO}

Más tardío es el uso de pronto, procedente del adjetivo latino promptus, - $a$, -um, con el valor de 'dispuesto, listo, presto, diligente, inclinado', cultismo de empleo escaso, pero constante, en época medieval, muchas veces aún con la grafía latinizante prompto.

En documentos notariales de los siglos X-XII redactados en latín, aparece el adjetivo promptus, especialmente en sintagmas fijados por el uso, en construcciones de ablativo absoluto en que acompaña a determinados sustantivos como cor, animus, mens, voluntas: prompto corde, promto animo, 'con corazón dispuesto, con ánimo/ propósito dispuesto"7, etc. En el siglo XIV, el CORDE y el CDH documentan este

la etimología propuesta por Menéndez Pidal (1977: 810) que parte directamente de privado, aunque en este caso el proceso de deslizamiento semántico resulta más complicado. Entiende que desde 'privado' se llega al valor de 'en particular', 'especialmente', 'fácilmente', y de este a 'en seguida, pronto' (Corominas 1980-1991, vol. IV: $655 b-656 a)$

4 Para que se produzca la transformación del sustantivo en adverbio tenemos que partir de entornos en que funcione como complemento circunstancial, lo que puede explicarse por su uso en ablativo, o tras preposición que indique su función, como sugiere Corominas (1980-1991: I, 88b), que propone partir de la secuencia a aína, todavía presente en Berceo, ad ahina (Milagros 278, sólo en el ms. I, copia de Ibarreta realizada en el siglo XVIII sobre un manuscrito del XIII, hoy perdido), con fusión de la preposición con la $a$ - inicial del sustantivo.

5 Aina aún tiene cierta supervivencia dialectal en áreas como Asturias, Murcia o Canarias (Alvar y Pottier 1983: 338, $\S$ 231.1, García Soriano 1932: 123, s. v. aínas, Academia de la Llingua Asturiana, 2001: 154, Corrales, Corbella y Álvarez 1992: 58, s. v. aína). Sánchez Sevilla (1928: 159, observación recogida en Corominas 1980-1991, vol. I: 88b) señalaba su pervivencia en la primera mitad del siglo XX, con la pronunciación áinas en Cespedosa de Tormes, y Corominas (1980-1991; vol. I: 88b) se refiere también a las locuciones compuestas no tan aína(s), por ainas en Bogotá. El CORDE, el CREA y el CDH muestran un puñado de ejemplos en textos del XIX y XX, generalmente en contextos en que se recoge o imita el habla rústica y vulgar.

6 Del uso adjetivo de presto con el valor de 'rápido' pudo pasarse al adverbial 'rápidamente', y de ahí al temporal 'pronto, enseguida'. Aparte del acercamiento del valor adjetivo al adverbial en los usos como complemento predicativo, es también posible pensar en la conversión directa de adjetivos en adverbios, procedimiento que probablemente fue frecuente en el latín vulgar (Hummel 2000: 476, 2008: 129), y que sería una continuación del procedimiento habitual en latín de utilizar la forma neutra singular del adjetivo como adverbio, y dio lugar al uso como adverbios de alto, bajo, claro, etc., a los que se unieron otros adjetivos (Grandgent 1928: 55; Väänänen 1963: 168-169; Lapesa 2000: 263-265, Hummel 2000: 454-461).

El CORDE recoge 4 veces la secuencia prompto corde, 4 veces prompa mente, 2 veces prompta voluntate, una prompto animo y una prompta devotione en documentos notariales fechados entre 945 y 1181. 
adjetivo, usado en escasa medida, tanto con la grafía pronto como prompto, con el significado de 'dispuesto, inclinado' en textos castellanos y aragoneses. El empleo de pronto aumenta en el siglo XV, época en que es intenso el uso de latinismos, en algunas ocasiones coordinado con adjetivos de semejante valor semántico como inclinado, aparejado, expedito o presto, ya sea en masculino o en femenino:

Despues d'esti, Segerico fue creado rey de los godos, el qual non res menos, commo semblantment por el indicio de Dios fuesse prompto et enclinado a la paz, fue muerto de los suyos (Juan Fernández de Heredia, Traducción de la Historia contra paganos, de Orosio, 1376-1396, apud CDH)

Marabillandose el rrey dela facultad tan prompta $\&$ aparejada de fa(j)blar del Esopo: dize le. Traxiste me los maestros que han de hedificar la torre (Esopete historiado, a 1482, apud $\mathrm{CDH})$

vos rogamos afectuosamente que, por nuestro amor y por los dichos respectos, fagays reuocar los dichos guiages, que empachan la paga e solucion de las dichas deudas, y mandeys ministrar sobreseo assi prompta y expedita justicia ( «Fernando ruega al rey de Nápoles haga administrar justicia a Jofre Pardo»[Documentos sobre relaciones internacionales de los Reyes Católicos], 1489, apud CDH)

Leuis. y hoc. ue. omnis generis. liuiana o ligera cosa: o presta o pronta (Rodrigo Fernández de Santaella, Vocabulario eclesiástico, 1499, apud CDH),

y aparece también la construcción en pronto, preposición + sustantivo, que retoma la construcción latina in promptu $^{8}$; pero, aunque funcionaba igual que ella como complemento circunstancial, su valor cambia algo respecto al que tenía en latín, 'a la vista, a mano, a disposición', y pasa en muchas ocasiones a equivaler a 'rápidamente, (muy) pronto, enseguida, inmediatamente', como puede verse en ejemplos como :

Si ventura faz' mudanza/ el triste se torna ledo, en pronto o quedo a quedo*/ tornará su bienandanza (Gómez Pérez Patiño, a 1435, apud CDH)

Por ellos las mortandades/vyenen, señor, en las tierras;/por ellos hanbres y guerras,/ fundyçiones de çibdades;/que muchas son destruydas/y fechas ynabytables,/ algunas otras fundidas/ y en pronto convertidas/ en lagunas espantables (Gómez Manrique, Poesias, 1479, apud CDH).

En el primero de estos ejemplos, en pronto está coordinado disyuntivamente con la expresión antónima quedo a quedo "poco a poco, despacio'.

No obstante, el uso de pronto en el siglo XV aún era bajo, como refleja el hecho de que Nebrija recoja en su Diccionario latino-español (1492) el término latino promptus: "Promptus.a.um.por cosa apercibida", mientras que no aparece pronto en el Vocabulario español-latino (1494). Y a pesar del incremento de su empleo a lo largo

El sustantivo promptus, -us solo se usaba en latín en la locución in promptu. Del mismo modo, pronto solo es término de preposición en esta construcción en el español medieval.

9 En unos pocos casos, y más en el español clásico que en el medieval, en pronto aparece con el valor de 'a la vista, a mano": "Y siendo necessario para el effecto de la dicha entrega lo siguiente, que el conuento de san felipe notificandossele esta peticion y aucto de V.m., se obligue en forma a tener estos bienes apreciados siempre en pronto y de manifiesto si acasso le mouieren o mouiere pleyto alguno" (Inventario y tasación de los bienes de Sebastiana Bocángel, 1630, apud CORDE). 
del siglo XVI, aún sigue sin tener una entrada en el Tesoro de la lengua castellana o española (1611) de Covarrubias, primer diccionario monolingüe del español. No obstante, sí aparece recogido en algunas obras lexicográficas del siglo XVI, como podemos ver en Nieto y Alvar (2007: VIII, 7980b), a partir del Vocabulario de las dos lenguas toscana y castellana de Cristóbal de Las Casas (Sevilla, 1570), que presenta una entrada para prompto y otra encabezada por presto o prompto ${ }^{10}$, y a partir de ahí en una amplia serie de diccionarios bilingües que recogen la equivalencia de pronto en otras lenguas.

El empleo del adjetivo pronto va creciendo a lo largo de los siglos XVI y XVII, conservando el valor de 'inclinado, dispuesto, presto', y, sigue apareciendo con cierta frecuencia en coordinación con su cuasi-sinónimo presto para aumentar el énfasis de la construcción. Resulta también significativo un ejemplo de Bocángel en el que aparece presta adjetivando a prontitud, y pronta a presteza, en un juego de construcciones cruzadas equivalentes:

Otro, que se hacía verdugo y secutor de justicia de Júpiter, como que estaba pronto para ejecutar lo que le mandase (Fray Bartolomé de las Casas, Apologética historia sumaria, 1527-1550, apud $\mathrm{CDH}$ )

Trae alas en los piés, con que denota

La presta prontitud, pronta presteza,

Con que ha de obedecerse el Evangelio (Bartolomé Cairasco de Figueroa, Obra poética, c. $1590-1610$, apud CDH)

Y entre ellas la primera y principal es, que el Encomendero que recibe del Rey la Encomienda, le prometa y jure fidelidad, especial servicio, y vasallage por esta merced, y estár presto y pronto con armas y cavallo, para militar y pelear por él contra cualesquier enemigos (Juan de Solórzano y Pereira, Política indiana, 1648, apud $\mathrm{CDH})$.

Por supuesto, desde que comienza a documentarse el adjetivo pronto comienza también a documentarse el adverbio de modo prontamente. Aunque no es un adverbio de tiempo, cuando se parte del valor de 'rápido' que podía presentar pronto se crea un adverbio celerativo, con el significado de 'rápidamente', que en alguna medida se aproxima al valor temporal. Puede aparecer también coordinado con prestamente y otros adverbios de modo:

requieren esta vez por todas que aceptéis los dichos finales ofrecimientos por la libertad de los dichos señores hijos de Francia por el bien de la paz universal y libertéis la persona de nuestro muy Santo Padre y también paguéis prontamente y sin más dilación lo que al dicho Rey mi soberano debéis (Alonso de Santa Cruz, Crónica del Emperador Carlos V, c. 1550, apud CDH).

\footnotetext{
Nieto y Alvar (2007: VIII, 7980b) recogen también su uso en dos obras lexicográficas de finales del XV: El Universal vocabulario en latín y romance (1490) de Alonso de Palencia, y el Vocabulario eclesiástico (1499) de Rodrigo Fernández de Santaella; pero en estos casos no aparece como lema de una entrada (de hecho en ambas obras las entradas corresponden a palabras latinas), sino en la equivalencia en castellano de la explicación dada en primer término en latín, en el caso de Palencia (en el que ya aparece el término promptus en el texto latino que luego se glosa), o en la equivalencia castellana de un término latino en el caso de Santaella.
} 
Hábito es cierta cualidad que dispone y habilita y determina la potencia donde se asienta, para que obre prompta y presta y fácilmente y con delectación y alegría (Fray Bartolomé de las Casas, Apologética historia sumaria, 1527-1550, apud CDH).

\section{Surgimiento DE LOS USOS ADVERBiALES DE PRONTO}

Desde las primeras apariciones de pronto hasta la segunda mitad del siglo XVIII, este término aparece utilizado de un modo casi exclusivo como adjetivo, no como adverbio, aunque el CORDE y el CDH recogen algunos testimonios de su uso adverbial desde el siglo $\mathrm{XV}^{11}$ :

por mucho que el esfuerço y maña le defendiese, el que más se tardaua más pronto muerto caya (Crónica incompleta de los Reyes Católicos, 1469-1476, ed. de Julio Puyol, Academia de la Historia (Madrid), 1934)

Quel alcalde con el procurador de pecheros vayan a ver lo del soto de Mohed, que defiende el secretario, e tome la sentençia e la execute e ponga en la posesión al procurador de la Villa e que se le haga saber por los dichos Luzon e Pedro de Monçon que lo provean pronto (Libro de Acuerdos del Concejo Madrileño, 1498-1501, ed. de Carmen Rubio Pardos; Rosario Sánchez González; Carmen Cayetano Martín, Ayuntamiento de Madrid (Madrid), 1982).

Sin embargo, la revisión de los pasajes a la vista de las lecturas de los originales nos muestra que se trata de malas transcripciones.

En el ejemplo de la Crónica incompleta del los Reyes Católicos, la consulta del manuscrito original muestra claramente la lectura presto en este pasaje ${ }^{12}$. La consulta del facsímil del original del Libro de Acuerdos del Concejo Madrileño, muestra igualmente la lectura presto ${ }^{13}$.

Los ejemplos que he podido comprobar de los siglos XVI, XVII e incluso de la primera mitad del XVIII también correspondían a malas lecturas. Así, por ejemplo, el CORDE recoge con cierta frecuencia el uso de pronto como adverbio de tiempo en la Historia general y natural de las Indias (1535-1557) de Gonzalo Fernández de

11 Aunque los mismos ejemplos están fichados en CORDE y CDH, preferimos en este caso y los siguientes el uso de CORDE, que señala la página en que se encuentra el ejemplo concreto en la edición utilizada, lo que permite su más fácil localización y, cuando es preciso, la verificación de la exactitud del ejemplo con el cotejo del original o facsímil del mismo.

12 En la edición de Julio Puyol, que ficha el CORDE, se lee efectivamente pronto. Hemos consultado el manuscrito original, que se halla en la Real Academia de la Historia (signatura 9-467 actualmente, no 12-3-4 como daba Puyol (1934: n. 1), procedente de la colección Salazar donde tenía la signatura G-20). Se trata de un manuscrito, tal como lo describe Puyol, con letra de finales del XV o principios del XVI, sin título, con letrero manuscrito en caracteres góticos en la tapa anterior en que se lee Cronica del Rey don Enrique 4. Se ha tachado en el título Enrique 4 y se ha escrito en letra cursiva del XVII Fernando y Doña Ysabel. Según Puyol, consta de 96 hojas, con los folios sin numerar. En la actualidad puede verse una numeración posterior a lápiz en el ángulo superior derecho del folio. El pasaje en cuestión se halla en el folio 89r, columna b. En la línea 6 se lee "presto muerto caya". En presto aparece abreviada la sílaba re mediante una trazo consistente en una pequeña línea sobre la $p$, que en la parte superior gira hacia la izquierda. Las letras psto se leen con claridad (la $\int$ es alta). La abreviatura comentada se usa en otros casos para $r+$ vocal. Por ejemplo, en el folio $88 \mathrm{v}^{\circ}$ columna a, vemos de esta manera abreviado prieto y principes.

13 Consulta hecha en microficha en el Archivo de la Villa el 8-5-2015. Como en el caso anterior, la palabra se halla abreviada como psto, en este caso con raya superpuesta sobre la $p$ y la $s$. Las confusiones esporádicas al resolver la abreviatura pueden deberse a que tanto presto como pronto comienzan y terminan del mismo modo ( $p r-t o)$, y a la tendencia espontánea de los editores modernos a usar el adverbio pronto. 
Oviedo. Siguiendo la edición de Juan Pérez de Tudela Bueso (Madrid, Atlas, 1992) ofrece, entre otros, pronto en el siguiente pasaje:

tienen jaolas de madera, donde ponen a engordar indios para los matar e comer después cuando les paresce que están bien en carnes, como se hace con los puercos en Castilla; e para que engorden más pronto, quiébranles los ojos.

Sin embargo, en la edición en línea de la Biblioteca Virtual Miguel de Cervantes ${ }^{14}$, reproducción digital de la edición de Amador de los Ríos (Madrid, Imprenta de la Real Academia de la Historia, 1855), se lee claramente presto en ese lugar.

El siguiente pasaje de Brito ofrece también un ejemplo de pronto como adverbio de tiempo:

Espero de acabar bien pronto esta fortaleza (Carta de Antonio Brito al Rey de Portugal sobre algunos sucesos en la India, 1523, Madrid, Imprenta Nacional, 1837, apud CORDE),

pero la carta de Brito al rey de Portugal que ficha el CORDE no corresponde al original de 1523, sino al extracto publicado por el editor del XIX, D. J. B. Muñoz, sobre un original portugués.

Entre los ejemplos de pronto como adverbio en el siglo XVII que recoge el CORDE hallamos, por ejemplo, el siguiente de Castillo Solórzano:

Sírvase vuestra merced de hacer presentación a su señora destos servicios, para que pronto, en su tribunal, alcancen el premio que merecen (Alonso de Castillo Solórzano, La niña de los embustes, Teresa de Manzanares, 1632, apud CORDE),

pero, consultado el original en la edición digital de la Biblioteca Nacional, en este pasaje se lee:

Siruase v.m. de hazer presentacion à su señora destos seruicios, para que puestos en su tribunal alcancen el premio que merecen.

Se trata por tanto de una errata clara. En el original no hay ningún adverbio de tiempo en este pasaje.

En la Historia del Nuevo Mundo de Bernabé Cobo recoge el CORDE cuatro casos de pronto, que efectivamente se hallan en la edición que digitaliza, de Marcos Jiménez de la Espada (Sevilla, Sociedad de Bibliófilos Andaluces, 1890-1893). Los dos primeros son los siguientes:

Es esta fruta muy dulce y huele á algalia, mas pierde pronto el olor, que por cierto es muy suave, y si lo conservara mucho tiempo, fuera de gran estimación (Bernabé Cobo, Historia del Nuevo Mundo, 1653, apud CORDE)

andaban unos pajarillos marinos poco mayores que gorriones, á bandadas, dando vueltas volando por encima del mar, y en dando una vuelta, se asentaban en un barco; vi que á

\footnotetext{
$<$ http://bib.cervantesvirtual.com/servlet/SirveObras/12604391997041537421624/ima0150.htm>.
} 
un vuelo que dieron muy á raíz del agua, se quedó uno asentado sobre ella revoleteando como que con violencia le tuvieren asido; llamé pronto á un muchacho que allí cerca estaba, y le hice entrase á cogerlo, que no distaba mucho de tierra (Bernabé Cobo, Historia del Nuevo Mundo, 1653, apud CORDE),

pero, comprobada su lectura en el facsímil del manuscrito del XVII digitalizado por la Universidad de Sevilla ${ }^{15}$, hallamos en el primer caso presto y en el segundo de presto. Muy probablemente en los otros dos casos nos hallamos también ante una modernización, que sustituye presto por pronto, debida al editor de finales del XIX, época en que ya pronto ha generalizado su empleo en detrimento de presto. Por la misma razón, resultan cuando menos dudosos otros ejemplos de la segunda mitad del XVII de pronto como adverbio que recoge el CORDE a través de ediciones de la segunda mitad del XIX, como sucede con el Diario de noticias de 1677 a 1678 de Juan Antonio de Valencia (Madrid, Imprenta de Miguel Ginesta, 1877), los Avisos (1654-1658) de Jerónimo de Barrionuevo (ed. de Antonio Paz y Meliá, Imprenta de M. Tello, Madrid, 1892-93) o las cartas (1678-1679) de Juan de Austria (ed. de Eugenio de Ochoa, Madrid, Rivadeneira, 1870).

Para la primera mitad del XVIII, el CORDE recoge el siguiente ejemplo de Torres Villarroel:

- Sé poco o nada de lo que me preguntas -respondí pronto-. Aparécete tú, cuando tú quisieres o Dios te lo mandare, a algún freile *o caballero de tu hábito; que éste te responderá con fundamento (Visiones y visitas de Torres con Don Francisco de Quevedo por la corte, 1717-1728, apud CORDE),

donde cabría la posibilidad de entender pronto como adverbio o como adjetivo en uso predicativo, pero la concordancia en género que vemos en otros pasajes semejantes en textos de la época nos indica claramente que se trata de un adjetivo:

Una Dama se excusó/De asistir, diciendo pronta:/Yo no gusto de ensalada,/Salpicon, ni pepitoria (Tomás de Iriarte, Poesías varias, 1730-1791 apud CORDE).

En otro pasaje su uso sería claramente adverbial:

Debe hacerse este matrimonio, luego que el agua esté hecha, y no se debe esperar más que ad súmmum dos horas, porque se desvanece pronto la virtud de este espíritu (Torres Villarroel, La suma medicina o piedra filosofal, 1726, Barcelona, Edicomunicación 1997, apud CORDE),

pero en la edición de 1726 se lee indudablemente presto $^{16}$, alterado por el editor moderno.

También encontramos un uso adverbial de pronto en la Poética de Luzán:

El duque de Alba le hizo su secretario. Dejando el servicio de aquel Grande, se casó en Madrid y enviudó pronto (La Poética o reglas de la poesía en general y de sus principales especies, 1737-1789, apud CORDE),

$<$ http://fondosdigitales.us.es/fondos/libros/2423/11/historia-del-nuevo-mundo-por-el-padre-bernabe-cobo-de-lacompania-de-jesus/>.

16 Hemos comprobado la lectura del pasaje a través de la edición digital del texto de 1726 de la BNE: < http://bdh-rd bne.es/viewer.vm?id=0000082679\&page $=1>$ 
pero no corresponde al texto de 1737 , sino que se halla en los añadidos de la edición de 1789, cuya autoría, como señala Sebold (1977: 64), es muy difícil de precisar.

Los diccionarios bilingües que incorporan este término desde Cristóbal de las Casas (1570) hasta Andree (1725) recogidos en Nieto y Alvar (2007) ofrecen siempre adjetivos en las equivalencias italianas, latinas, inglesas, francesas y alemanas para pronto en estas lenguas. Y el Diccionario de Autoridades (Real Academia Española 1737: 402a, apud NTLLE) incluye pronto como adjetivo y sustantivo, no como adverbio.

En la segunda mitad del siglo XVIII sí se encuentran ya casos claros e indudables, y cada vez más frecuentes, de pronto utilizado como adverbio de tiempo. El primer ejemplo del XVIII recogido en el CORDE sería el siguiente:

habiéndose podido entender un poco, porque el indio hablaba en castellano, con mucho gusto lo acarició, y le dijo que se viniese con él que pronto lo pondría en Buenos Aires (Viaje que hizo el "San Martín", desde Buenos Aires al Puerto de San Julián, 1752-1755, apud CORDE),

pero puede resultar dudoso porque la primera edición de este texto es de 1837 , aunque se refiera a sucesos acaecidos entre 1752 y 1755 , por lo que no sabemos si hay modificaciones debidas al editor del XIX. En cualquier caso, pocos años más tarde sí hallamos ejemplos seguros como los siguientes:

Pues, señor, sea lo que fuere,/lo que se ponga sea presto,/porque ya bailan, y pronto/querrán beber (Ramón de la Cruz, El reverso del sarao, 1766, apud CORDE)

Aqui tenemos, hace cuatro dias, uno de aquellos scirocos que matan las gentes, ó que, al menos, nos tuestan las cabezas, de modo que parecen calabazas secas: sino llueve pronto, irá subiendo de punto esta historia (Manuel de Azara, Cartas de Azara al ministro Roda en 1769, apud CORDE).

La aparición de los usos adverbiales de pronto podría deberse a un proceso de adverbialización del adjetivo iniciado a partir de empleos de carácter predicativo en que se aproxima al valor de un circunstancial, siguiendo un proceso paralelo, aunque más tardío, al que había experimentado el propio presto $^{17}$. Sin embargo, y sin negar que esto haya podido contribuir en alguna medida a la adquisición del valor adverbial, parece más probable que se haya producido una imitación de los usos adverbiales de presto por parte de pronto: sentido pronto como equivalente a presto en los usos adjetivos, se lleva más allá esa equiparación y se introduce también en los empleos adverbiales. De hecho, la influencia de presto sobre algunos usos de pronto se había dado probablemente antes, y explica el cambio de preposición en la locución en pronto, que, como ya vimos, desde el siglo XV era latinismo introducido con el valor de '(muy) pronto, en seguida'. A partir del siglo XVI, encontramos la variante de pronto que se generaliza en el XVIII, y que probablemente cambia la preposición por influencia de la locución de presto, que con el mismo valor encontramos desde el siglo XV, y con especial intensidad en los

Así lo interpreta, por ejemplo, Azofra (2014: 397). 
siglos XVI y XVII, para decaer en el XVIII precisamente cuando de pronto incrementa notablemente su empleo ${ }^{18}$. La locución de presto tal vez se crease siguiendo el modelo formal de otras locuciones como de repente o de súbito (Rodríguez Molina 2014: 786), que empiezan a documentarse casi al mismo tiempo que de presto, aunque el valor de de presto era el de adverbio temporal, variante formal del simple presto $^{19}$, no tenía el valor de marcar comienzo o cambio repentino de una acción como de súbito o de repente.

En el siguiente cuadro, podemos ver, basándonos en los datos del $\mathrm{CDH}$, el uso de las construcciones en pronto, de pronto y de presto entre los siglos XV (cuando comienzan a documentarse) y $\mathrm{XX}^{20}$. Damos el número total de ocurrencias y el número de textos en que los ficha el $\mathrm{CDH}$, así como su frecuencia relativa de empleo en palabras por millón ${ }^{21}$ :

Cuadro 1. Uso de las construcciones en pronto, de pronto, de presto, siglos XV-XX (CDH)

\begin{tabular}{|c|c|c|c|c|c|c|}
\hline & XV & XVI & XVII & XVIII & XIX & $\mathrm{XX}$ \\
\hline $\begin{array}{c}\text { en } \\
\text { pronto }\end{array}$ & $\begin{array}{l}40 / 28 \\
1,6 \text { p. mill }\end{array}$ & $\begin{array}{l}11 / 11 \\
0,19 \text { p. mill }\end{array}$ & $\begin{array}{l}3 / 3 \\
0,07 \text { p. mill }\end{array}$ & $0^{22}$ & 0 & $\begin{array}{l}1^{23} \\
0,005 \text { p. mill. }\end{array}$ \\
\hline $\begin{array}{c}\mathrm{de} \\
\text { pronto }\end{array}$ & 0 & $\begin{array}{l}9 / 6 \\
0,16 \text { p. mill }\end{array}$ & $\begin{array}{l}10 / 10 \\
0,24 \text { p. mill }\end{array}$ & $\begin{array}{l}94 / 61 \\
5,1 \text { p.mill }\end{array}$ & $\begin{array}{l}2086 / 414 \\
40,5 \text { p. mill }\end{array}$ & $\begin{array}{l}15416 / 2379 \\
75,3 \text { p. mill }\end{array}$ \\
\hline $\begin{array}{l}\text { de } \\
\text { presto }\end{array}$ & $\begin{array}{l}23 / 17 \\
0,9 \text { p. mill }\end{array}$ & $\begin{array}{l}935 / 270 \\
16,2 \text { p. mill. }\end{array}$ & $\begin{array}{l}263 / 110 \\
6,4 \text { p. mill }\end{array}$ & $\begin{array}{l}7 / 6 \\
3,8 \text { p. mill }\end{array}$ & $\begin{array}{l}24 / 17 \\
0,47 \text { p. mill }\end{array}$ & $\begin{array}{l}38 / 12 \\
0,19 \text { p. mill }\end{array}$ \\
\hline
\end{tabular}

18 A partir del valor originario de 'enseguida, apresuradamente, súbitamente', se usa en determinados contextos en los que, sin perder ese valor, puede interpretarse también con el de 'de repente', como en "Pero salteóle de pronto un intensíssimo sentimiento a Critilo que le apretó el coraçón hasta exprimirle por los ojos" (Baltasar Gracián, El Criticón segunda parte, 1653, apud CORDE); "El rey luego que vio fixado aquel terrible cometa en el cielo de su palacio, aunque ignoraba el contenido de la escritura por entonces, los latidos de su conciencia que ya no podía disimular, coadyuvados con los tristes recuerdos de su difunto padre que de pronto le asaltaron a su memoria, le comenzaron a pronosticar alguna fatalidad" (Fray Joaquín Bolaños, La portentosa vida de la muerte, México, 1792, apud CORDE), lo que permitirá su desplazamiento a este valor. La acepción 'de repente' la recoge el NTLLE por primera vez en el Diccionario Nacional de Joaquín Ramón Domínguez ( ${ }^{5} 1853$; pero está ya en ${ }^{2} 1847$ : 1430a, que se puede consultar en la edición en línea de la BNE, <http://bdh-rd.bne.es/viewer.vm?id=0000002387\&page=1>), y la Academia la incluirá en la edición de 1884. En extensas áreas de Hispanoamérica, tanto de pronto como de repente llegan a alcanzar el valor de 'probablemente, quizás', uso registrado en Asociación de Academias de la Lengua Española (2010: 1772b) en Nicaragua, Colombia, Ecuador y Perú, aunque su área de empleo debe ser más extensa. Rojas Gallardo (2008) estudia el uso de de repente en el español de Chile, y el proceso que ha llevado a esta expresión a funcionar como "marcador de modalidad epistémica atenuador de la fuerza argumentativa" ('quizás'), y señala que un proceso paralelo se ha dado con de pronto.

19 Y ese es el único valor que da el diccionario académico de 1803, el primero que recoge esta locución (NTLLE).

20 En las secuencias en pronto y de pronto sumamos los casos en que aparecen con las grafías pronto y prompto. En el caso de de pronto hemos restado un ejemplo de siglo XVI del anónimo Viaje a la Tercera, hecho por el comendador de Chaste, por tratarse realmente de un texto escrito originariamente en francés en el XVI, y traducido al español en el XVIII, por lo que no es representativo del período en que lo incluye el CDH, y hemos restado también 10 ejemplos del siglo XVIII en que las secuencias de pronto/prompto que recoge el CDH no corresponden a la locución adverbial, sino a la secuencia de + pronto + sustantivo, del tipo de pronto remedio, hombre de prompto ingenio, etc.

21 Para calcular el número de ocurrencias por millón de palabras, tenemos en cuenta el número de palabras registradas en CDH en cada siglo. Siglo XV: 25.543.793; XVI: 57.886.242; XVII: 40.895.054; XVIII: 18.278.984; XIX: 51.465.530; XX: 204.747.641.

22 El CDH recupera una secuencia en pronto para el siglo XVIII, pero no se trata de la locución adverbial, sino de un sintagma preposicional en el que pronto es adjetivo que acompaña a un sustantivo: "en pronto vuelo".

${ }_{23}$ El CDH recupera dos ejemplos de la secuencia en pronto; pero uno de ellos, "En Pronto Mayor" del argentino 
Vemos en este cuadro cómo la disminución del empleo de en pronto corre paralela al crecimiento de de pronto, y este a su vez arranca aún tímidamente en el siglo XVI, momento en el que hallamos el mayor auge del empleo de de presto, que probablemente motiva la introducción de esta variante, que se convierte en mayoritaria en el XVII frente a en pronto, y aumenta rápidamente su uso en el XVIII, a costa de de presto que queda reducida a un uso marginal. El pequeño índice de empleo que mantiene de presto en los siglos XIX y XX, en textos escritos y básicamente cultos, obedece probablemente a arcaización formal a partir de la forma general de pronto del español moderno. Tal vez la existencia de la locución adverbial de pronto sea un segundo factor que, junto a la imitación del comportamiento de presto, adverbio y adjetivo, favoreciese el paso de pronto a usos adverbiales. La proximidad, casi identidad significativa, entre pronto y presto en los usos adjetivos es algo que de hecho queda reflejado en la lexicografía preacadémica: Cristóbal de las Casas da para el italiano pronto la equivalencia castellana, prompto, presto (Casas 1579: 110v) y presenta la doble entrada sinonímica presto ò prompto, para el que da la equivalencia italiana presto (226r). El Diccionario de Autoridades (Real Academia Española 1737: 369a, apud NTLLE), en la definición de las dos acepciones de presto en usos adjetivos, utiliza el término pronto: "PRESTO, TA. adj. Pronto, diligente, liberal y ligéro en la execucion de alguna cosa", "Significa tambien aparejado y pronto para executar alguna cosa". El empleo de pronto como adverbio, que como vimos, documentan el CORDE y el CDH poco después de mediados del XVIII, lo reflejará la lexicografía académica con medio siglo de retraso, en la edición de 1817: "pronto, adv. m. lo mismo que prontamente", aunque, como señala Corominas (1980-91: IV, 660a), prontamente "no es lo mismo que presto, luego". Resulta curioso que el DRAE catalogue en 1817 a pronto como adverbio de modo y no de tiempo (tal vez por la equivalencia que propone con un adverbio en -mente), cuando el propio adverbio prontamente al que remite está catalogado como adverbio de tiempo. La descripción como "adv. m." se mantuvo hasta la edición de 1917. A partir de la de 1936 sí se cataloga como adverbio de tiempo, aunque sin cambiar la definición que propuso ya en 1899: "Presto, prontamente". En la edición de 2014 se dan las acepciones adverbiales, encabezadas simplemente por la indicación $a d v$, sin precisar de qué tipo de adverbio se trata, práctica en cualquier caso general en los diversos tipos de adverbios, pues, como la Real Academia avisa en la introducción de la vigesimotercera edición, "en los adverbios se han eliminado las subespecificaciones «t.», de tiempo; «m.», de modo; «cant.», de cantidad, etc. ${ }^{24 "}$ (RAE-ASALE 2014: XLV). Se elimina la definición "presto, prontamente" y se incluye como quinta acepción (primera de las adverbiales) "temprano (// en las primeras horas del día)", que no estaba recogida antes, y también una octava como adverbio de modo celerativo "rápidamente (// con celeridad)"25. Sorprende, sin embargo, que no aparezca una acepción para el valor 'en un plazo breve', que es el primer valor adverbial que recoge el DEA, y al que en ediciones anteriores del diccionario académico se apuntaba con la equivalencia "presto, prontamente" eliminada ahora.

Macedonio Fernández, no corresponde a la construcción que estudiamos. El otro ejemplo, que aparece en la prensa venezolana: "apuntó que son simples molestias y que en pronto podrá reaparecer al campo" (El Nacional, 19/01/1997), aunque lo hemos contabilizado, parece una errata, tal vez por cruce de pronto con la construcción en breve.

24 No obstante, sí aparecen específicamente marcados los llamados adverbios demostrativos (adv. dem.), como aquí o ahora, que antes venían marcados como adverbios de lugar o tiempo.

25 Sobre los usos celerativos de pronto v. Rodríguez Molina (2014: 754-55). 
Es cierto que el surgimiento de los usos adverbiales de pronto y su rápido crecimiento se producen cuando presto ya casi ha dejado de usarse como adjetivo, pero esos usos residuales serían suficientes para desatar la equiparación de los dos elementos y el paso de pronto a los usos adverbiales. Presto, utilizado como adverbio de tiempo, tiene un importante crecimiento a finales de la Edad Media, y ese crecimiento va acompañado de un descenso, hasta su casi desaparición, del uso del adverbio medieval aína, y posteriormente también de los usos de presto como adjetivo. Si observamos los datos que ofrece el $\mathrm{CDH}$ sobre el empleo de presto en la segunda mitad del XV, podemos observar un rápido crecimiento de su uso, manteniendo todavía una frecuencia alta de usos adjetivos, mientras que, en la segunda mitad del XVII, aunque el empleo de presto sigue siendo alto, los usos adjetivos son claramente marginales.

Analizando los usos de presto registrados en el $\mathrm{CDH}$ en los períodos comprendidos entre 1451-1470 y 1481-1490 podemos observar cómo se produce un fuerte crecimiento del empleo de presto, acompañando al creciente empleo de sus usos adverbiales ${ }^{26}$. Así, para el primer período, 1451-1470, documenta el CDH 246 ejemplos de presto, de los que, dejando aparte 12 casos que corresponden a formas del verbo prestar, 100 corresponden a usos adjetivos ${ }^{27}$ y 134 a usos adverbiales ${ }^{28}$. Para el segundo período, 1481-1490, el CDH recoge 833 ejemplos de presto, de los que 220 corresponden a usos adjetivos y 558 a usos adverbiales ${ }^{29}$; hay además 4 ejemplos de la locución adverbial de presto, 35 casos que corresponden al verbo prestar, y 16 proceden de textos escritos en latín. Vemos, por una parte, cómo el porcentaje de empleos de los usos adverbiales frente a los adjetivos aumenta: en el primer período hallamos 1,3 usos adverbiales por cada uso adjetivo, en el segundo de 2,5; por otra parte, aumenta también la intensidad de empleo de presto en la lengua. Para el período 1451-1470 hay 2.729.201 palabras en los textos recogidos en el CDH, lo que implica un empleo de presto de 90,1 ocurrencias por millón de palabras, mientras que para el período 1481-1490 hay 4.440.822 palabras en los textos fichados, lo que implica una frecuencia de 187,57 usos de presto por millón de palabras, es decir, más del doble que en el primer período. Si nos fijamos en el empleo de presto en la segunda mitad del XVII (1651-1700), en el que el CDH recoge 955 casos sobre un corpus de 7.429 .780 palabras, vemos una intensidad de uso

26 Comparamos los usos de la forma presto usada como adverbio y como adjetivo en masculino singular, que es la forma que puede confundirse con el adverbio. Naturalmente, si sumamos los casos en que se usan las formas para el singular femenino y plural masculino y femenino (presta, prestos, prestas) crece la proporción de usos adjetivos. Sin embargo, los datos obtenidos son lo suficientemente representativos para el objetivo que queremos estudiar: el creciente empleo de presto en esta época, y el aumento de la proporción de usos adverbiales.

27 Muchos de los usos adjetivos de presto, tanto en este período como en el período 1481-90, se deben al frecuente empleo de la fórmula estáles presto de + infinitivo en el lenguaje administrativos de los libros de acuerdos, especialmente los del concejo madrileño. Esta secuencia es igualmente frecuente en plural están/son prestos de + infinitivo.

28 Si tenemos en cuenta la ocurrencias de presto adjetivo que recoge el CDH también en las formas de masculino plural (prestos, 99), femenino singular (presta, 23. El CDH recoge 37 veces la forma presta en este período, pero 6 corresponden al adverbio de modo escrito con el sufijo -mente separado: presta mente, y en 8 ocasiones es forma del verbo prestar) y masculino plural (prestos 99), los usos adjetivos, 235, superan ampliamente a los adverbiales.

29 Si sumamos las ocurrencias de presto adjetivo que recoge el CDH también en las formas de masculino plural (prestos, 183), femenino singular (presta, 63. El CDH recoge 129 veces la forma presta en este período, pero en 63 ocasiones forma parte del adverbio de modo presta mente, escrito con sus formantes separados, y en 3 es forma del verbo prestar) y femenino plural (prestas, 17. El CDH recoge 19 veces la forma presta, pero en dos ocasiones son formas verbales en latín), los empleos de presto adjetivo llegan a más del doble, 483. Aun así, su aparición es inferior a la del adverbio presto. 
algo inferior a la del período 1481-1490 (128,5 palabras por millón), y un clarísimo predominio de los usos adverbiales ( 889 empleos adverbiales y solo 23 adjetivos ${ }^{30}$, aparte de una errata por preso, 22 usos del verbo prestar y 20 de la locución adverbial de presto). Si observamos el desarrollo del empleo de presto desde la segunda mitad del XV, momento en que se produce el primer fuerte crecimiento de este término, especialmente debido a su incremento en usos adverbiales, en los que desplaza a aína, vemos un importante aumento en la primera mitad del XVI, y el mantenimiento de unos niveles altos de uso, pero con una tendencia decreciente desde la segunda mitad del XVI hasta la segunda mitad del XVII. A partir del XVIII se da una fuerte caída de su empleo, hasta quedar en niveles muy bajos desde la segunda mitad del XIX. El lento descenso de presto desde la segunda mitad del XVI a la segunda mitad del XVII apenas va acompañada de un crecimiento de los usos de pronto $^{31}$, sin embargo, desde el siglo XVIII, especialmente desde la segunda mitad, coincidiendo con la aparición de los usos adverbiales de pronto, el rápido declive de presto sí va acompañado de un también rápido crecimiento de pronto. Podemos observar tanto el movimiento de ascenso y posterior descenso de los usos de presto como el aumento de pronto, primero muy lento y acelerado desde la segunda mitad del XVIII en el siguiente cuadro, en el que vemos los usos de presto y pronto divididos por períodos de 50 años, excepto los dos últimos, que desglosamos en períodos de 25 :

Cuadro 2. Frecuencia de presto $^{32}$ y pronto (1451-2000). Datos de CDH

\begin{tabular}{|l|l|c|c|c|c|}
\hline Período & $\begin{array}{c}\text { № de palabras } \\
\text { registradas en } \mathrm{CDH} / \\
\mathrm{n} \text { - documentos }\end{array}$ & $\begin{array}{c}\text { № de } \\
\text { ejemplos } \\
\text { de presto }\end{array}$ & $\begin{array}{c}\text { Usos de presto } \\
\text { por millón de } \\
\text { palabras }\end{array}$ & $\begin{array}{c}\text { oo de } \\
\text { ejemplos de } \\
\text { pronto } 33 / \mathrm{n} \text { - } \\
\text { documentos }\end{array}$ & $\begin{array}{c}\text { Usos de pronto } \\
\text { por millón de } \\
\text { palabras }\end{array}$ \\
\hline $1451-1500$ & $16.834 .158 / 4050$ & $2434 / 392$ & 144,6 & $47 / 30$ & 2,8 \\
\hline $1501-1550$ & $22.983 .095 / 3.238$ & $4997 / 494$ & 217 & $111 / 52$ & 4,8 \\
\hline $1551-1600$ & $34.903 .147 / 4.369$ & $5438 / 672$ & 155,8 & $175 / 80$ & 5 \\
\hline $1601-1650$ & $33.465 .274 / 5.064$ & $5.046 / 671$ & 150,8 & $272 / 22$ & 8,1 \\
\hline $1651-1700$ & $7.429 .780 / 1.244$ & $955 / 141$ & 128,5 & $95 / 55$ & 12,7 \\
\hline $1701-1750$ & $7.717 .082 / 283$ & $309 / 64$ & 40 & $258 / 94$ & 33,4 \\
\hline $1751-1800$ & $10.561 .902 / 2.201$ & $601 / 153$ & 56,9 & $758 / 291$ & 71,8 \\
\hline $1801-1850$ & $13.758 .896 / 1.618$ & $548 / 99$ & 39,8 & $2.509 / 438$ & 182,4 \\
\hline $1851-1900$ & $37.706 .634 / 1.879$ & $301 / 115$ & 8 & $9.105 / 700$ & 241,4 \\
\hline $1901-1950$ & $42.734 .066 / 5.006$ & $464 / 121$ & 10,9 & $9.425 / 1.137$ & 220,6 \\
\hline $1951-1975$ & $30.904 .685 / 1.852$ & $179 / 79$ & 5,8 & $5.570 / 435$ & 180,2 \\
\hline $1976-2000$ & $131.108 .890 / 104.524$ & $322 / 196$ & 2,5 & $12.237 / 4.332$ & 93,3 \\
\hline
\end{tabular}

30 Si tenemos en cuenta las formas de femenino singular (presta, 12. El CDH recoge 52 ocurrencias de presta en este período, pero la mayoría corresponden a formas del verbo prestar), masculino plural (prestos, 8) y femenino plural (prestas, 4. EL CDH recoge 7 veces prestas, pero en 3 ocasiones son formas del verbo prestar), llegamos a un total de 47 ocurrencias de presto adjetivo en todas su formas, lo que sigue estando muy lejos de la intensidad de aparición de presto como adverbio.

31 De hecho, el uso de pronto en esta época es incluso algo inferior al que refleja la tabla, pues, como ya hemos visto, entre el XV y el XVIII el CORDE y el CDH incluyen usos adverbiales de pronto que no están en los originales a través de modificaciones de ediciones modernas.

32 El CDH recoge un número mayor de ejemplos de la forma presto en los distintos períodos que hemos establecido, pero hemos eliminado aquellos en que corresponden a formas del verbo prestar (con las grafías presto y prestó), más frecuentes en textos modernos.

33 Hasta el período 1751-1800 sumamos los ejemplos que aparecen con la grafía pronto y los que aparecen con la grafía prompto. A partir del período 1801-1850 el CDH solo recoge ejemplos con la grafía pronto. 
Como hemos señalado, en el período comprendido entre finales del XV y finales del XVII, el uso de pronto crece muy poco, a pesar de que hay un descenso en los usos adjetivos de presto. Esto es probablemente debido a que son otros adjetivos, que parcialmente coinciden con valores de presto o pronto, como aparejado, inclinado y, más tarde, dispuesto, los que crecen en sus empleos. En el siglo XVIII, pronto sí va a crecer en gran medida, primero en sus usos adjetivos, ya en la primera mitad, y con mucha más intensidad a partir de la segunda mitad cuando se comienzan a sumar sus empleos como adverbio. A partir de ahí, por una parte se producirá un incremento notable de su empleo, que queda prácticamente estabilizado desde la segunda mitad del XIX; por otra, una progresiva disminución de sus usos como adjetivo, en beneficio de otros como listo, dispuesto o preparado. Si estudiamos los ejemplos de pronto que recoge el $\mathrm{CDH}$ en la segunda mitad del XVIII y primera del XIX observamos un notable crecimiento, tanto de su empleo en términos absolutos, como de los usos adverbiales, como muestra el análisis de los ejemplos recogidos en los períodos comprendidos entre 1751-1800 y 1801-1825 $5^{34}$. Para el primer período, dejando aparte los usos de pronto como sustantivo y su aparición en las locuciones al pronto, de pronto, por el/lo pronto, por de pronto, e incluso tres ejemplos en que aparece en un parlamento en italiano, encontramos 330 ejemplos de usos adjetivos y 344 de usos adverbiales. Hay también 6 ejemplos de la locución conjuntiva temporal tan pronto como. Además, puede señalarse que en las primeras décadas de este período hay un predominio de los usos adjetivos, frente a las dos últimas en que predominan los usos adverbiales ${ }^{35}$. En el período 1801-1825, dejando aparte los mismos casos que hemos comentado antes, encontramos 132 casos de usos adjetivos por 301 de usos adverbiales ${ }^{36}$. Se ha pasado de un equilibrio entre los empleos adjetivos y adverbiales de pronto en la segunda mitad del XVIII a un uso claramente mayoritario (más del doble de ejemplos) de los usos adverbiales en el primer cuarto del siglo XIX, y esa tendencia sigue en aumento en época posterior, aunque su uso disminuye algo en la segunda mitad del XX. Este es un fenómeno claramente panhispánico, que lleva al establecimiento de pronto como adverbio predominante para la expresión de la cercanía temporal en todos los países de lengua española. ${ }^{37}$

34 Para el período 1751-1800 hemos pedido al CDH que recupere las secuencias pronto y prompto.

35 También pueden observarse claras preferencias por el empleo de pronto como adjetivo o adverbio en diversos autores. Así, por ejemplo, José Francisco de Isla lo utiliza casi siempre como adjetivo en su Fray Gerundio de Campazas (1758), en tanto que Iriarte (El señorito mimado, 1787, La señorita malcriada, 1788) o Antonio José Cavanilles (Observaciones sobre la historia natural, geografia, agricultura, población y frutos del reyno de Valencia, II, 1797) lo emplean casi siempre en su función de adverbio.

36 Por lo que se refiere a la forma pronto. Por supuesto, los usos adjetivos crecen teniendo en cuenta las formas para el femenino y el plural hasta alcanzar 304 ocurrencias (132 de pronto, 115 de prontos, 42 de pronta y 15 de prontas), lo que representa el mismo índice de empleo que el uso adverbial. Entre los usos adverbiales incluimos los casos de pronto que aparecen en correlaciones comparativas (con valor distributivo) tan pronto...como, pero no aquellos en que forma parte de la locución conjuntiva de posterioridad inmediata tan pronto como, aunque esta se forma sobre el uso adverbial.

37 La intensidad del empleo actual de pronto es casi idéntica en España y en Hispanoamérica. Aunque, al pedirle las estadísticas para el siglo XX, los datos que ofrece el CDH muestran un uso aparentemente menor en España que en los restantes países de habla española (56,5 usos por millón de palabras en España, frente a 64,36 en el área andina, 80 en la chilena o hasta 131 en las Antillas), esto se debe a que la estadística está hecha sobre el total de palabras para cada país, desde los primeros textos hasta la actualidad, y no se limita a los datos del siglo XX. Por otro lado, el peso de los textos americanos antes del siglo XVIII, época en que el uso de pronto apenas despunta, es muy escaso. Si tenemos en cuenta sólo los datos del siglo XX, tenemos 17.357 usos de pronto en textos españoles, que presentan 
Cuando pronto pasa a tener eminentemente usos adverbiales, básicamente equivalentes a los que antes tenía presto, lo empezaremos también a encontrar formando parte de locuciones conjuntivas como tan pronto como, en las que antes intervenía presto (tan presto como), y en época medieval aína (tan aína como):

tan pronto como con las señales de paz tornaron los ánimos, mis Capitanes se abstuvieron de toda hostilidad (Carta del Arzobispo al General Guillermo Draper, Filipinas, 1762, apud $\mathrm{CDH})^{38}$.

Entre el valor de cercanía temporal, 'en un plazo breve', y el también temporal de 'con anticipación al momento oportuno, esperado o convenido' existe una cierta proximidad en determinados contextos, pues lo que sucede en un plazo breve sucede con frecuencia con anticipación a un momento esperado, que es el valor fundamental de temprano ${ }^{39}$; de ahí que en determinados contextos se produzca una neutralización de estos valores, y en el español clásico y del siglo XVIII encontremos con frecuencia el adverbio presto en relación con tarde, en idénticos contextos que temprano, especialmente en secuencias del tipo presto o tarde, y en el español medieval en los mismos contextos se podía hallar aína. Cuando pronto comienza a usarse como adverbio de tiempo equivalente a presto lo hallaremos también en estos contextos ${ }^{40}$ :

Estos tristes compromisos, harto lo dice la historia, conducen pronto ó tarde á inexplicables solidaridades (Crónica esterior [La Ciencia Cristiana, octubre de 1877], apud $\mathrm{CDH}$,

pero a partir de estos contextos, pronto llegará a una equiparación más extensa con temprano que la que había tenido presto. Así hallamos, al menos desde la transición de la Edad Media al Renacimiento, la construcción temprano para, con el valor de 'antes del momento oportuno o esperable para algo'.

pero como quando esto se acabó aún fuese temprano para se recoger ... (Alonso de Santa Cruz, Crónica de los Reyes Católicos, 1491-1516, apud CDH).

Y según Azofra (2009: 23), desde el siglo XV el uso de temprano se extiende para referirse a las primeras horas del día (o de la noche, como adecuadamente se recoge en la definición del DRAE):

E deque vino la mañana salieron bien temprano dela posada (Libro llamado Infancia Salvatoris. BNM I-2400, a. 1493, apud CDH)

un total de 124.952 .886 palabras; y 10.344 en textos americanos, que presentan un total de 79.720.003 palabras; lo que supone 138,9 usos de pronto por millón de palabras en textos españoles, y 129,75 en textos americanos.

38 El CORDE y el CDH registran ejemplos anteriores de la secuencia tan pronto como, pero no constituyendo una locución conjuntiva, sino en secuencias puramente comparativas.

39 El adjetivo temprano procede del latín vulgar temporanus, que desde San Agustín aparece con la acepción 'que se hace a tiempo' (Corominas 1980-1991: 487a) y ha sufrido también un proceso de adverbialización en época preliteraria.

40. Aunque el adverbio pronto aparece en relación con tarde desde la segunda mitad del XVIII, el CORDE y el CDH no recogen la construcción pronto o tarde hasta la segunda mitad del XIX. 
E, llegada la noche, Eneas se echó temprano, porque madrugar pudiese (Enrique de Villena, Traducción y glosas de la Eneida. Libros I-III, 1427-28, apud CDH),

valores ambos en los que no se encuentra presto, pero que sí alcanzará pronto, y que documenta el CDH desde el segundo cuarto del XIX:

Me contento, pues, con la pobre isla de Ítaca, por más que sea pequeña y pobre, harta gloria me cabrá si llego a reinar en ella con justicia, valor y piedad, aunque recelo que siempre será demasiado pronto para llegar a ocupar el trono (Mariano Antonio Collado, Traducción de las aventuras de Telémaco seguidas de las de Aristonoo de Fénelon, 1843, apud CDH)

Pues a la ejecución, dijo el padre; mañana pronto monto a caballo y me voy a ver a Morfina y su buena madre (Braulio Foz, Vida de Pedro Saputo, 1844, apud CDH),

aunque el Diccionario de la Real Academia no incluirá la primera acepción hasta 1970: "6. Con anticipación al momento fijado, dispuesto o acostumbrado; con sobra de tiempo", y la segunda 'en las primeras horas del día' no se recogía aún en la vigésima segunda edición de 2001 . No obstante sí se daba ya en el artículo enmendado que la Academia ofrecía como avance de la vigésima tercera edición en la aplicación en línea ( $<$ http://lema.rae.es/drae/?val=pronto $>$ ), y efectivamente ha sido recogido en la edición de 2014 (RAE-ASALE 2014: 1795b).

\section{USOS DE PRONTO COMO SUSTANTIVO. INTEGRACIÓN EN DIVERSAS LOCUCIONES}

Desde el siglo XVIII, encontramos también usos sustantivos de pronto, que surgen probablemente a través de la reinterpretación de la secuencia de pronto, modificación, como ya vimos, de la más antigua en pronto por influencia analógica de de presto, que siempre tuvo de un modo exclusivo un valor adverbial, como secuencia formada por preposición + sustantivo. En un primer momento, el sustantivo pronto viene a utilizarse con el significado de 'momento', como parecen mostrar ejemplos como:

atendiendo el Comandante que aquella partida que despachó la noche antes ya habría llegado a la acción, y que oyendo los tiros era natural pensasen los enemigos tenían a todo Buenos Aires sobre sí, y que con este motivo tirasen a huir, dispuso en aquel pronto desparramar en pelotones indios y cristianos (Juan Antonio Hernández, Diario que el capitán, don Juan Antonio Hernández ha hecho de la expedición contra los indios teguel, Argentina, 1770, apud CDH)

Al pronunciar estas últimas palabras puede libertar una mano; con el mayor [58] denuedo coge la espada a Guillelmo, y en un pronto, sin poderse éste precaver, se la entra por el pecho y cae sin aliento (Ignacio García Malo, Voz de la naturaleza. Memorias o anécdotas curiosas e instructivas, 1787 - 1803, apud CDH).

En este último caso, el contexto en que aparece permite interpretar pronto como 'momento, instante breve', pero también como 'movimiento súbito' ${ }^{4}$, valor que llega

$\overline{41}$ El matiz de acción o movimiento súbito pudo estar influenciado por el propio significado de la locución de pronto. 
a adquirir rápidamente, especialmente con el matiz de 'movimiento del ánimo', al que debió llegarse muy pronto, pues ya aparece recogido en Autoridades ${ }^{42}$ (Academia 1737: 402a, apud NTLLE): "sust. movimiento repentino de alguna pasión: como le dió un pronto y tomo la capa para salirse de casa.", definición a la que se añade en la novena edición, de 1843, "movimiento repentino de alguna pasión u ocurrencia inesperada" y se modifica en la décima edición de 1852 en "El movimiento repentino a impulsos de alguna pasión u ocurrencia inesperadas, como le dió un pronto y tomo la capa para salir de casa". En la decimonovena edición, de 1970, se añade una nueva acepción catalogada, como la anterior, de familiar: “4. fam. Ataque repentino y aparatoso de algún mal", procedente con toda probabilidad de un deslizamiento a partir de la acepción anterior.

También el significado 'momento' es el que aparece en la construcción por el pronto, que el CORDE y el CDH comienzan a registrar a partir de mediados del XVIII:

se bautizaron casi todos los indios adultos, por estar ya capaces para ello, habiendo desde los principios franqueado este sacramento el padre Fritz a los párvulos, que no necesitaban, por el pronto, la instrucción que aquéllos en los misterios de la fe (Jorge Juan, Noticias secretas de América, 1747, apud CDH)

y un poco más tarde se registra también por lo pronto ${ }^{43}$ :

Por esto le han escrito que se esté quieto, y le han enviado mil escudos, para que se socorra por lo pronto (José Nicolás de Azara, Cartas de Azara al ministro Roda en 1769, apud $\mathrm{CDH})$,

pero la construcción con el artículo masculino es, con mucho, más frecuente en la segunda mitad del XVIII y primera del XIX, lo que resulta lógico si pensamos en el origen señalado: locución prepositiva que contiene el lexema pronto reinterpretado como sustantivo masculino. De ahí que sólo se incluya en 1803, en el "Suplemento de algunas voces omitidas en el diccionario" (apud NTLLE) la variante por el pronto, que se define como "mod. adverbial. Interinamente, entretanto, provisionalmente". A partir de la segunda mitad del XIX, el empleo de por lo pronto alcanza e incluso rebasa la intensidad de empleo de por el pronto ${ }^{44}$. Esto se debe probablemente al hecho de que el progresivo abandono de pronto como sustantivo con el valor de 'momento' haría que no se sintiera motivada la construcción, que sí entra dentro de esquemas de construcción normales al utilizarse la variante con el artículo neutro, forma habitual de sustantivar un adjetivo. El crecimiento de la variante por lo pronto se ve reflejada en la inclusión de la doble posibilidad "por el, ó lo, pronto" en la duodécima edición del diccionario académico, de 1884.

42 Que en cambio no recoge la acepción de 'momento, instante'. También podría tomar ese valor a través de la reinterpretación de pronto como 'movimiento súbito, impulsivo' a partir del valor de 'súbitamente' que llega a adquirir la locución de pronto.

43 El ejemplo de por lo pronto de 1725 que recogen el CORDE y el CDH en el Correo del otro mundo de Torres Villarroel: "Sentí a este tiempo un ruido hacia los pies, y por lo pronto consentí que fuese alguna sabandija de las que criamos a nuestros pechos, que se arrimó a morderle los zancajos" no corresponde a la expresión que ahora estudiamos, sino que es una construcción con artículo neutro sustantivador del adjetivo, con el valor de 'por lo veloz'.

44 El CDH registra 61 ejemplos de por el pronto y 37 de por lo pronto en el período 1801-1850, y 110 ejemplos de por el pronto frente a 178 de por lo pronto en el período 1851-1900. 
La locución por de pronto, que el CORDE y el CDH documentan desde finales del siglo XIX:

No hay duda que son ciertas estas proposiciones; pero suprimiendo el cultivo del arroz, no sé si mejoraria el estado actual de las cosas. La propiedad por de pronto sería como es hoy de los que no habitan en Sueca (Antonio José Cavanilles, Observaciones sobre la historia natural, geografia, agricultura, población y frutos del reyno de Valencia, 1795, apud CDH),

parece alteración de por el pronto por influencia formal de la locución adverbial de pronto.

Igualmente se forma a partir del uso sustantivo de pronto con el valor de 'momento' la locución al pronto, que registran el CORDE y el CDH también hacia mediados del XVIII ${ }^{45}$ :

Anoche escribí sobre el punto de sueldos, y repito que me parece conveniente que al pronto se me envíen las listas (Simón de Anda y Salazar, Carta del Sr. Anda al P. Braña, 1763, apud CORDE),

aunque la locución no será recogida en la lexicografía académica hasta la undécima edición de 1869, en que se define, “AL PRONTO mod adv. Á PRIMERA VISTA", y en la duodécima edición de 1884 se modifica en "En el primer momento o a primera vista", que es la redacción que permanece en la actualidad.

\section{CONCLUSIONES}

Pronto es un adverbio temporal de formación y consolidación moderna, que se constituye en el elemento más importante para la expresión de la cercanía temporal o posterioridad próxima respecto a un punto cualquiera fijado por el hablante sin idea explícita de inmediatez (para este valor el elemento más destacado en el español moderno es el adverbio en seguidalenseguida, aunque pronto pueda ir reforzado por adverbios intensificadores como muy o bien si se quiere aumentar la idea de proximidad temporal). En principio, fue un adjetivo introducido por vía culta, que llega a arraigar y generalizar su uso y, como otros adjetivos, alcanza usos adverbiales, aunque en este caso probablemente su adverbialización se deba a una absorción de los usos adverbiales de presto, elemento con el que presentaba una proximidad significativa amplia en sus usos adjetivos. La consolidación del adverbio pronto junto con la también moderna consolidación de en seguida/enseguida lleva al establecimiento en el español moderno de un sistema de adverbios para la expresión de la posterioridad próxima diferente del que había existido en el español clásico, en el que el elemento principal para la expresión de la cercanía temporal era presto

\footnotetext{
El CORDE y el DDH recogen un ejemplo anterior, de 1730, de difícil interpretación, aunque parece significar 'en el momento': "ha de ser en la ocasion urgentíssima el recurso, y que lo era, y es oy actualmente, certissima la decadencia de facultades laycas, aunque consideraràn otros medios, siendo los que proponían en la circunstancia intratables, al pronto que la necessidad pedia, solo con una reserva para restituirse, si no estuvieran por otros medios compensados" (Francisco Máximo de Moya Torres y Velasco, Manifiesto universal de los males envejecidos que España padece, 1730, apud CDH). Otro que da de la Poética de Luzán (1737-1789) corresponde a las adiciones de 1789.
} 
(aunque sus usos no están siempre nítidamente diferenciados de los de posterioridad inmediata), que terminará siendo casi abandonado, excepto en usos retóricos o dialectales, y el elemento fundamental para la expresión de la posterioridad inmediata estaba constituido por luego, que, perdiendo el rasgo de inmediatez, lo que comienza a ser frecuente a partir del XVIII, pasará a indicar simple posterioridad en el español moderno, alcanzando un valor más o menos equivalente al de después.

\section{Siglas UTILIZADAS}

CDH: Corpus del Nuevo diccionario histórico V. Instituto de Investigación Rafael Lapesa de la Real Academia Española.

CORDE: Corpus Diacrónico del Español. V. Real Academia Española.

CREA: Corpus de Referencia del Español Actual. V. Real Academia Española.

DEA: Diccionario del español actual. V. Seco, Manuel, Olimpia Andrés y Gabino Ramos.

NTLLE: Nuevo tesoro lexicográfico del la lengua española. V. Real Academia Española.

RAE-ASALE: Real Academia Española-Asociación de Academias de la Lengua Española.

\section{OBRAS CITADAS}

Academia de la Llingua Asturiana. ${ }^{3}$ 2001. Gramática de la llingua asturiana. Uviéu: Academia de la Llingua Asturiana.

Alvar, Manuel y Bernard Pottier. 1983. Morfología histórica del español. Madrid: Gredos.

Asociación de Academias de la Lengua Española. 2010. Diccionario de americanismos. Madrid: Santillana.

Azofra Sierra, Elena. 2009. "Deixis adverbial temporal en la historia del español". Anuario de Lingüistica Hispánica XXV: 15-34.

Azofra Sierra, Elena. 2014. "Adverbios de tiempo. Demostrativos, comparativos y modotemporales", Concepción Company (dir.), Sintaxis histórica de la lengua española. Tercera Parte: Preposiciones, adverbios y conjunciones. Relaciones interoracionales, vol I. México: FCE-UNAM. 313-410.

Casas, Cristóbal de las. 1570. Vocabulario de las dos lenguas toscana y castellana, Sevilla: Francisco de Aguilar. Accesible en < http://books.google.es/books/about/Vocabulario de_las_dos_lenguas_toscana_y.html?id=XMOUHUbCJzkC\&redir_esc $=\mathrm{y}>$.

Corominas, Joan. 1980-1991. Diccionario crítico etimológico castellano e hispánico, con la colaboración de José A. Pascual. Madrid: Gredos, 6 vols.

Corrales Zumado, Cristóbal, Dolores Corbella Díaz y Ma Ángeles Álvarez Martínez. 1992. Tesoro lexicográfico del español de Canarias. Madrid: Real Academia EspañolaGobierno de Canarias. Conserjería de Educación, Cultura y Deportes.

García Soriano, Justo. 1932. Vocabulario del dialecto murciano. Madrid: Bermejo.

Grandgent, C. H. 1928. Introducción al latín vulgar. Madrid: Centro de Estudios Históricos.

Hummel, Martin. 2000. Adverbale und adverbialisierte Adjektive im Spanischen. Konstruktionen des Typs Los niños duermen tranquilos und María corre rápido. Tübingen: Narr.

Hummel, Martin. 2008. "La predicación secundaria en el habla oral informal de Chile". Estudios de Lingüistica de la Universidad de Alicante 22: 129-149.

Instituto de Investigación Rafael Lapesa de la Real Academia Española. 2013. Corpus del Nuevo diccionario histórico (CDH) [en linea]. <http://web.frl.es/CNDHE> [Consulta: marzo-abril 2016]. 
Lapesa, Rafael. 2000. "Sintaxis histórica del adjetivo calificativo no atributivo". Estudios de morfosintaxis histórica del español. Madrid, Gredos: 235-271.

Menéndez Pidal, Ramón. ${ }^{5}$ 1977. Cantar de Mio Cid. II. Vocabulario. Madrid: Espasa-Calpe.

Nieto, Lidio y Manuel Alvar. 2007. Nuevo Tesoro Lexicográfico del Español (s. XIV-1726). Madrid: Arco/Libros.

Real Academia Española. Nuevo tesoro lexicográfico del la lengua española $<\mathrm{http} / /$ buscon. rae.es/ntlle/SrvltGUIMenuNtlle?cmd=Lema\&sec=1.0.0.0.0.>.

Real Academia Española: Banco de datos (CORDE) [en línea]. Corpus diacrónico del español (CORDE). <http://corpus.rae.es> [consulta: mayo de 2015 y marzo-abril de 2016].

Real Academia Española: Banco de datos (CREA) [en línea]. Corpus de referencia del español actual. $<$ http://www.rae.es $>$ [consulta: mayo de 2015].

Real Academia Española-Asociación de Academias de la Lengua Española. 2009. Nueva gramática de la lengua española. Madrid: Espasa Libros.

Real Academia Española-Asociación de Academias de la Lengua Española. 2014. Diccionario de la lengua española, $23^{\mathrm{a}}$ ed., Edición del Tricentenario. Madrid: Espasa.

Rodríguez Molina, Javier. 2014. "Adverbios y locuciones adverbiales de manera”, Concepción Company (dir.), Sintaxis histórica de la lengua española. Tercera Parte: Preposiciones, adverbios y conjunciones. Relaciones interoracionales, vol I. México: FCE-UNAM. 733-937.

Rojas Gallardo, Darío. 2008. "Funciones actuales y evolución semántica de la locución de repente en el español de Chile”. Boletin de Filología XLIII: 207-237.

Sánchez Lancis, Carlos. 1990. Estudio de los adverbios de espacio y tiempo en el español medieval, tesis doctoral. Univeristat Autònoma de Barcelona. Accesible en red: $<\mathrm{http}: /$ tdx.cat/handle/10803/4893>.

Sánchez Sevilla, P. 1928. "El habla de Cespedosa de Tormes (en el límite de Salamanca y Ávila)”. Revista de Filología Española XV: 131-172.

Sebold, Rusell P. 1977. prólogo a Ignacio de Luzán, La poética. Reglas de la poesía en general $y$ de sus principales especies. Barcelona: Labor.

Seco, Manuel, Olimpia Andrés y Gabino Ramos. ${ }^{2} 2011$. Diccionario del español actual. Madrid: Aguilar.

Väänänen, Veikko. 1963. Introduction au latin vulgaire. Paris : Klincksieck. 